Proceedings of the 1999 IEEE

International Conference on Robotics \& Automation

Detroit, Michigan - May 1999

\title{
A Two Loops Direct Visual Control of Direct-Drive Planar Robots with Moving Target*
}

\author{
Rafael Kelly ${ }^{\dagger}$, Fernando Reyes ${ }^{\ddagger}$, Javier Moreno ${ }^{\dagger}$, and Seth Hutchinson§ \\ † División de Física Aplicada, CICESE, Apdo. Postal 2615, Adm. 1 \\ Ensenada, B. C., 22800, MEXICO \\ e-mail: rkelly@cicese.mx \\ ‡ Escuela de Ciencias de la Electrónica, Universidad Autónoma de Puebla \\ Puebla, Puebla, 72570, MEXICO \\ e-mail: freyes@kim.ece.buap.mx \\ $\S$ Department of Electrical and Computer Engineering \\ The Beckman Institute for Advanced Science and Technology \\ University of Illinois at Urbana-Champaign \\ Urbana, IL. 61801, USA
}

\begin{abstract}
This paper addresses the visual servoing of robot manipulators in fixed-camera configuration considering a moving target. We propose a control scheme consisting of two loops: an inner loop, which is a joint velocity controller, and an outer loop which is an image-based feedback loop. We present the stability analysis and the experimental evaluation on a two degrees of freedom direct-drive planar robot arm.
\end{abstract}

\section{Introduction}

Visual servoing of robot manipulators deals with the posture control of the robot end-effector frame either relative to the world coordinate frame or relative to a target object by using real-time visual information $[1,2]$. Two types of visual servoing configurations can be recognized nowadays: fixed-camera (or static camera) and camera-in-hand (or eye-in-hand). In this paper we address the fixed-camera approach to visual servoing of planar robot manipulators with a single camera but considering that the target posture

*Work partially supported by CONACyT-NSF grants No. 228050-5-C084A and IRI-9613737, project Perception Systems for Robots-CYTED and CONACyT I27218-A. is time varying. The visual control problem considered is a subclass of the stereo visual servoing with moving targets (see $[3,4,5,6,7,8,9,10]$ ). Previous efforts in visual servoing of fixed-camera robotic systems incorporating the full nonlinear robot dynamics in the analysis have been reported in $[11,12,13,14]$.

Hager [15] has categorized visual servoing systems according to several criteria. The first criterion is whether visual feedback is directly converted to joint torques or whether internal encoder feedback is used to implement a velocity servo in a hierarchical control arrangement. The former is referred to as direct visual servo while the latter to as look-and-move. Most of practical implemented system belong to the lookand-move variety.

In this paper we propose a visual servoing system consisting of two loops: an inner loop, which is a joint velocity servo, and an outer loop which is an imagebased feedback loop. Despite a velocity servo is used, this control scheme corresponds to direct visual servo because the control actions are the joint torques.

The originality of our approach partly relies on the fact that we show that a rigorous stability analysis for the look-and-move technique can be performed without neglecting the nonlinear robot dynamics but provided that an explicit asymptotic velocity controller is used.

Other properties of the proposed visual servo con- 
trollers are:

- "Endpoint-closed-loop" (ECL) system: The vision system provides both the target and endeffector postures defined in terms of observable features rigidly attached to them [15].

- Image-based: Define servoing errors directly from the camera image.

\section{Robotic system model}

The robotic system considered in this paper is composed by a planar robot manipulator and a vision system including a fixed camera as depicted in figure 1.

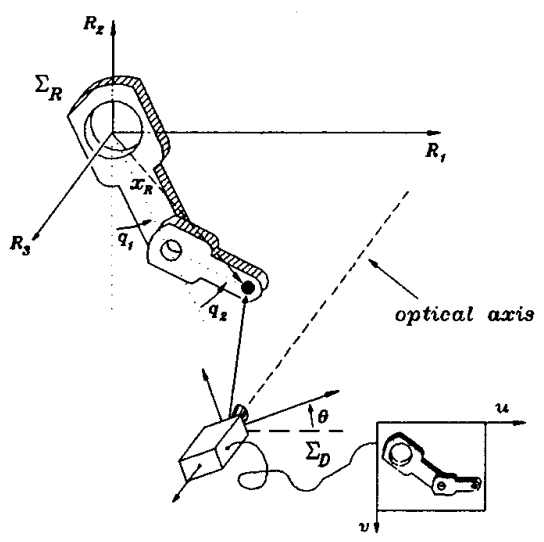

Figure 1: Robotic system.

\subsection{Robot dynamics}

In the absence of friction or other disturbances, the dynamics of a serial $n$-link rigid robot can be written as [16]:

$$
M(\boldsymbol{q}) \ddot{\boldsymbol{q}}+C(\boldsymbol{q}, \dot{q}) \dot{\boldsymbol{q}}+\boldsymbol{g}(\boldsymbol{q})=\boldsymbol{\tau}
$$

where $\boldsymbol{q}$ is the $n \times 1$ vector of joint displacements, $\dot{\boldsymbol{q}}$ is the $n \times 1$ vector of joint velocities, $\tau$ is the $n \times 1$ vector of applied torques, $M(q)$ is the $n \times n$ symmetric positive definite manipulator inertia matrix, $C(\boldsymbol{q}, \dot{\boldsymbol{q}})$ is the $n \times n$ matrix of centripetal and Coriolis torques, and $\boldsymbol{g}(\boldsymbol{q})$ is the $n \times 1$ vector of gravitational torques.

Let $\Sigma_{R}=\left\{R_{1}, R_{2}, R_{3}\right\}$ be a $3 \mathrm{D}$ right-hand Cartesian frame attached to the robot base. Consider the planar robot manipulator with two degrees of freedom moving in the plane $R_{1}-R_{2}$ as depicted in figure 1 . The direct kinematics gives the position $x_{R} \in \mathbb{R}^{2}$ of the robot tip (end-effector) with respect to the robot coordinate frame $\left(x_{R 3}=0\right)$ in terms of the joint positions $q \in \mathbb{R}^{2}$ :

$$
\boldsymbol{x}_{R}=\boldsymbol{f}(\boldsymbol{q})
$$

where $f: \mathbb{R}^{2} \rightarrow \mathbb{R}^{2}$. The so-called analytical Jacobian matrix $J_{A}(\boldsymbol{q}) \in \mathbb{R}^{2 \times 2}$ of the robot is defined from direct kinematics as

$$
J_{A}(\boldsymbol{q})=\frac{\partial \boldsymbol{f}}{\partial \boldsymbol{q}}
$$

\subsection{Vision system model}

A TV camera (CCD type) stationary with respect to $\Sigma_{R}$ provides images of the whole robot workspace, including the robot end-effector and any other visible object as shown the Figure 1.

The position of the camera frame with respect to $\Sigma_{R}$ is denoted by $o_{C}=\left[o_{C_{1}}, o_{C_{2}}, o_{C_{3}}\right]^{T}$. The camera frame may possess a rotation $\theta$ around axis $R_{3}$.

The image of the scene on the CCD is digitalized and transferred to the computer memory and displayed on the computer screen. We define the two dimensional computer image (screen) coordinate frame $\Sigma_{D}=\{u, v\}$. The origin of $\Sigma_{D}$ is attached at the upper left corner of the computer screen while the axes $u$ and $v$ are selected parallel to the screen rows and columns respectively.

As defined previously, $\boldsymbol{x}_{R}$ represent the position of the robot tip with respect to the robot frame $\Sigma_{R}$. This description depends on the joint position $q$. The description of such a point in the computer image (screen) frame $\Sigma_{D}$ denoted by $\left[\begin{array}{ll}u & v\end{array}\right]^{T}$ defines a mapping called the vision system model which includes a perspective projection and a rigid body transformation $[4,13]$ :

$$
\left[\begin{array}{l}
u \\
v
\end{array}\right]=\boldsymbol{h}(\boldsymbol{q})
$$

with

$$
\begin{aligned}
& \boldsymbol{h}(\boldsymbol{q})=\left[\begin{array}{cc}
-\alpha_{u} & 0 \\
0 & \alpha_{v}
\end{array}\right]\left[\frac{\lambda}{\lambda-o_{C_{3}}} R(\theta)^{T}\right. \\
& \left.\left[\left[\begin{array}{l}
x_{R_{1}}(\boldsymbol{q}) \\
x_{R_{2}}(\boldsymbol{q})
\end{array}\right]-\left[\begin{array}{l}
o_{C_{1}} \\
o_{C_{2}}
\end{array}\right]\right]+o_{I}\right]+\left[\begin{array}{l}
u_{0} \\
v_{0}
\end{array}\right], \\
& R(\theta)=\left[\begin{array}{ll}
\cos (\theta) & -\sin (\theta) \\
\sin (\theta) & \cos (\theta)
\end{array}\right],
\end{aligned}
$$

where $\alpha_{u}>0, \alpha_{v}>0$ are the scale factors in pixels $/ \mathrm{m}$, and $u_{0}, v_{0}$ denote the pixel position of the geometric center of plane CCD with respect to the system $\Sigma_{D}$, $R(\theta) \in S O(2)$ is the rotation matrix which represents the orientation of the camera with respect to the world 
frame $\Sigma_{R}, \lambda>0$ is the focal length of the camera, and the position of the intersection of the optical axis with respect the geometric center of the plane CCD is denoted by $\boldsymbol{o}_{I}$.

\subsection{Control aim}

The robot task is specified in the image plane in terms of image features corresponding to observable points rigidly attached to the robot tip and target object (one point attached to each one). It is assumed that the target object resides in the plane $R_{1}-$ $R_{2}$ but its position with respect to the robot and camera frames is unknown. Let $\left[\begin{array}{ll}u_{d} & v_{d}\end{array}\right]^{T}$ the description with respect to the computer image (screen) frame $\Sigma_{D}$ of the target image feature corresponding to the attached point. Hereafter, $\left[\begin{array}{ll}u_{d} & v_{d}\end{array}\right]^{T}$ will be referred as the desired image feature vector.

The control problem it to design a controller to compute the applied torques $\tau$ in such a way that the image feature $\left[\begin{array}{ll}u & v\end{array}\right]^{T}$ corresponding to the point attached to the robot tip reaches the desired image feature $\left[\begin{array}{ll}u_{d} & v_{d}\end{array}\right]^{T}$ of the point attached to the target object. This formulation can be equivalently stated as driving the robot tip in such a way that the corresponding image feature $\left[\begin{array}{ll}u & v\end{array}\right]^{T}$ reaches asymptotically a moving point $\left[\begin{array}{ll}u_{d}(t) & v_{d}(t)\end{array}\right]^{T}$ into the computer image (screen) frame.

The image feature error is defined as

$$
\left[\begin{array}{l}
\tilde{u} \\
\tilde{v}
\end{array}\right]=\left[\begin{array}{l}
u_{d}-u \\
v_{d}-v
\end{array}\right]
$$

therefore, the control aim is to assure that $\lim _{t \rightarrow \infty}\left[\begin{array}{ll}\tilde{u}(t) & \tilde{v}(t)\end{array}\right]^{T}=\mathbf{0} \in \mathbb{R}^{2}$, at least for initial conditions $\left[\begin{array}{ll}\tilde{u}(0) & \tilde{v}(0)\end{array}\right]^{T}$ and $\dot{\boldsymbol{q}}(0)$ sufficiently small.

In order to the control problem be solvable, we assume that there exists a joint motion $\boldsymbol{q}_{d}(t) \in \mathbb{R}^{2}$ such that

$$
\begin{aligned}
{\left[\begin{array}{l}
u_{d}(t) \\
v_{d}(t)
\end{array}\right] } & =\left[\begin{array}{cc}
-\alpha_{u} & 0 \\
0 & \alpha_{v}
\end{array}\right]\left[\frac{\lambda}{\lambda-o_{C_{3}}} R(\theta)^{T}\right. \\
& {\left.\left[\left[\begin{array}{l}
x_{R_{1}}\left(\boldsymbol{q}_{d}(t)\right) \\
x_{R_{2}}\left(\boldsymbol{q}_{d}(t)\right)
\end{array}\right]-\left[\begin{array}{l}
o_{C_{1}} \\
o_{C_{2}}
\end{array}\right]\right]+o_{I}\right]+\left[\begin{array}{l}
u_{0} \\
v_{0}
\end{array}\right] . }
\end{aligned}
$$

\section{A two loops direct visual servo}

The rationale behind the proposed control scheme is based on the look-and-move approach where the robot inner velocity loop provided by the robot manufacturer is invoked and used by an user defined imagebased outer loop. However, in our approach we obviate the unrealistic assumption that a ideal velocity loop is available to maintaining the joint (or endeffector) velocities tracking time varying desired velocities computed by the image-based outer loop.

\subsection{Velocity inner loop}

Inverse dynamics control is a well known textbook technique for motion control of robot manipulators [16]. Besides this application, it is also useful to deal with other control aims such as joint velocity control. In this situation an effective velocity controller is given by

$$
\begin{aligned}
\boldsymbol{\tau} & =M(\boldsymbol{q})\left[\ddot{\boldsymbol{q}}_{d}+K_{v} \dot{\tilde{\boldsymbol{q}}}+K_{p} \boldsymbol{z}\right]+C(\boldsymbol{q}, \dot{\boldsymbol{q}}) \dot{\boldsymbol{q}}+\boldsymbol{g}(\boldsymbol{q})(8) \\
\dot{\boldsymbol{z}} & =\dot{\tilde{\boldsymbol{q}}}
\end{aligned}
$$

where $\dot{\boldsymbol{q}}_{d}$ stands for the desired joint velocity, $\dot{\tilde{\boldsymbol{q}}}=$ $\dot{\boldsymbol{q}}_{d}-\dot{\boldsymbol{q}}$ denotes the joint velocity error, and $K_{p}$ and $K_{v}$ are $n \times n$ symmetric positive definite matrices. The closed-loop system is obtained by substituting the controller (8)-(9) into the robot dynamics (1). This yields the linear system

$$
\frac{d}{d t}\left[\begin{array}{c}
\boldsymbol{z} \\
\dot{\tilde{\boldsymbol{q}}}
\end{array}\right]=\left[\begin{array}{c}
\dot{\tilde{\boldsymbol{q}}} \\
-K_{v} \dot{\tilde{\boldsymbol{q}}}-K_{\mathrm{p}} \boldsymbol{z}
\end{array}\right]
$$

and because the positive definiteness of $K_{p}$ and $K_{v}$, then it is globally asymptotically stable and therefore the velocity error $\dot{\tilde{q}}$ vanishes, i.e., $\lim _{t \rightarrow \infty} \dot{\tilde{q}}(t)=\mathbf{0}$.

\subsection{Image-based outer loop}

Most of look-and-move visual servoing schemes are based on the assumption that manipulators act as ideal positioning devices where the actual joint or endeffector velocity tracks exactly desired velocity. Under this philosophy, image-based visual servoing systems are designed according to the following ideas.

The key point is to notice that from (4), the relationship between the time derivative of the image feature vector and the joint velocity is given by

$$
\left[\begin{array}{c}
\dot{u} \\
\dot{v}
\end{array}\right]=\underbrace{\frac{\partial \boldsymbol{h}(\boldsymbol{q})}{\partial \boldsymbol{q}}}_{J(\boldsymbol{q})} \dot{\boldsymbol{q}}
$$

where $J(\boldsymbol{q})$ is a Jacobian matrix. From the look-andmove optics, the robotic system is described by (10) where the system output is the feature vector $\left[\begin{array}{ll}u & v\end{array}\right]^{T}$ and its input is the joint velocity $\dot{\boldsymbol{q}}$.

By assuming that $J(q)^{T} J(q)$ is nonsingular, then the pseudo-inverse $J(\boldsymbol{q})^{\dagger}=\left[J(\boldsymbol{q})^{T} J(\boldsymbol{q})\right]^{-1} J(\boldsymbol{q})^{T}$ 
exists $^{1}$. Under such consideration, the desired joint velocity denoted by $\dot{\boldsymbol{q}}_{d}$ to be computed by the imagebased outer loop is usually given by

$$
\dot{\boldsymbol{q}}_{d}=J(\boldsymbol{q})^{\dagger}\left[\left[\begin{array}{l}
\dot{u}_{d} \\
\dot{v}_{d}
\end{array}\right]+K\left[\begin{array}{l}
\tilde{u} \\
\tilde{v}
\end{array}\right]\right]
$$

where $K$ is a symmetric positive definite matrix. It is clear now that owing to assumption of exact velocity tracking $\dot{\boldsymbol{q}} \equiv \dot{\boldsymbol{q}}_{d}$ and substituting (11) into (10) we get

$$
\frac{d}{d t}\left[\begin{array}{l}
\tilde{u} \\
\tilde{v}
\end{array}\right]=-K\left[\begin{array}{l}
\tilde{u} \\
\tilde{v}
\end{array}\right]
$$

which implies $\lim _{t \rightarrow \infty}[u(t) v(t)]^{T}=0$. Unfortunately, this conclusion has been obtained under the unrealistic assumption of perfect velocity tracking.

\subsection{Velocity plus image-based feedback}

In this subsection the assumption on perfect velocity tracking is relaxed. Indeed, the proposed visual servoing system is composed by the asymptotic velocity controller (8)-(9) together with the image-based controller (11). The latter provides the joint desired velocity $\dot{\boldsymbol{q}}_{d}$ from which the joint desired acceleration $\ddot{\boldsymbol{q}}_{d}$ is obtained. More specifically, the desired acceleration is given by

$$
\begin{aligned}
\ddot{\boldsymbol{q}}_{d}= & \frac{d}{d t}\left[J(\boldsymbol{q})^{\dagger}\right]\left[\left[\begin{array}{c}
\dot{u}_{d} \\
\dot{v}_{d}
\end{array}\right]+K\left[\begin{array}{c}
\tilde{u} \\
\tilde{v}
\end{array}\right]\right] \\
& +J(\boldsymbol{q})^{\dagger}\left[\left[\begin{array}{c}
\ddot{u}_{d} \\
\ddot{v}_{d}
\end{array}\right]+K\left[\begin{array}{c}
\dot{\tilde{u}} \\
\dot{\tilde{v}}
\end{array}\right]\right]
\end{aligned}
$$

It is important to remark that the image feature rate is involved in the computation of $\ddot{\boldsymbol{q}}_{d}$ through $\left[\begin{array}{ll}\dot{\tilde{u}} & \dot{\tilde{v}}\end{array}\right]^{T}$. Since $\left[\begin{array}{ll}\dot{u} & \dot{v}\end{array}\right]^{T}$ may be difficult to measure, then for implementation purpose it can be obtained invoking the Jacobian via (10) [17].

The closed-loop system is obtained by substituting the desired velocity and acceleration from (11) and (12) into the velocity controller (8) and (9) and then into the robot dynamics (1). The overall closed--loop equation is given by

$\frac{d}{d t}\left[\begin{array}{c}\boldsymbol{z} \\ \dot{\tilde{\boldsymbol{q}}} \\ \tilde{\boldsymbol{u}} \\ \tilde{\boldsymbol{v}}\end{array}\right]=\left[\begin{array}{cccc}0 & I & 0 & 0 \\ -K_{p} & -K_{v} & 0 & 0 \\ 0 & 0 & k_{11} & k_{12} \\ 0 & 0 & k_{21} & k_{22}\end{array}\right]\left[\begin{array}{c}\boldsymbol{z} \\ \dot{\tilde{\boldsymbol{q}}} \\ \tilde{u} \\ \tilde{v}\end{array}\right]+\left[\begin{array}{c}0 \\ 0 \\ J(\boldsymbol{q}) \dot{\tilde{\boldsymbol{q}}}\end{array}\right]$

where $k_{i j}$ are the entries of matrix $K$.

\footnotetext{
${ }^{1}$ In our application of a two degree of freedom $\operatorname{arm}(n=2)$, the pseudo-inverse becomes the inverse matrix.
}

Notice that $z$ and $\dot{\tilde{q}}$ evolves independently of the image feature error $\left[\begin{array}{ll}\tilde{u} & \tilde{v}\end{array}\right]^{T}$. Because the linear and asymptotically stable nature of the equation for these variables we get the conclusion of $\lim _{t \rightarrow \infty} \dot{\tilde{q}}(t)=0$ in an exponential manner.

On the other hand, assuming that the Jacobian $J(\boldsymbol{q})$ is bounded, then the term $J(\boldsymbol{q}) \dot{\tilde{\boldsymbol{q}}}$ vanishes exponentially. Since this term drives the linear and asymptotically stable system related to $\tilde{u}$ and $\tilde{v}$ in (13), then we finally have the conclusion $\lim _{t \rightarrow \infty}[\tilde{u}(t) \tilde{v}(t)]^{T}=0$.

\subsection{Discussion}

The simplest way for computation of the desired joint velocity $\dot{\boldsymbol{q}}_{d}$ is given by (11). Notwithstanding, it produces large desired velocity when the image feature error $\left[\begin{array}{ll}\tilde{u} & \tilde{v}\end{array}\right]^{T}$ is large too. This may be undesirable because possible saturation of the actuators and overshoot. An alternative fashion to compute the desired joint velocity is given by

$$
\dot{\boldsymbol{q}}_{d}=J(\boldsymbol{q})^{\dagger}\left[\left[\begin{array}{l}
\dot{u}_{d} \\
\dot{v}_{d}
\end{array}\right]+K \tanh \left[\Gamma\left[\begin{array}{l}
\tilde{u} \\
\tilde{v}
\end{array}\right]\right]\right]
$$

where $K$ and $\Gamma$ are diagonal positive definite matrices, and $\tanh (x)=\left[\tanh \left(x_{1}\right) \cdots \tanh \left(x_{n}\right)\right]^{T}$ for any vector $\boldsymbol{x} \in \mathbb{R}^{n}$.

The corresponding desired acceleration $\ddot{\boldsymbol{q}}_{d}$ is obtained by differentiating (14) which leads to

$$
\begin{gathered}
\ddot{\boldsymbol{q}}_{d}=\frac{d}{d t}\left[J(q)^{\dagger}\right]\left[\left[\begin{array}{l}
\dot{u}_{d} \\
\dot{v}_{d}
\end{array}\right]+K \tanh \left[\Gamma\left[\begin{array}{c}
\tilde{u} \\
\tilde{v}
\end{array}\right]\right]\right] \\
+J(q)^{\dagger}\left[\left[\begin{array}{l}
\ddot{u}_{d} \\
\ddot{v}_{d}
\end{array}\right]+K \Gamma \operatorname{sech}^{2}\left[\Gamma\left[\begin{array}{c}
\tilde{u} \\
\tilde{v}
\end{array}\right]\right]\left[\begin{array}{c}
\dot{\tilde{u}} \\
\tilde{\tilde{v}}
\end{array}\right]\right] .
\end{gathered}
$$

By invoking similar arguments than in the analysis of (13), above expressions for the desired velocity and acceleration yield $\lim _{t \rightarrow \infty}[\tilde{u}(t) \tilde{v}(t)]^{T}=\mathbf{0}$.

\section{Experimental set-up}

We have designed and built at CICESE Research Center a planar direct drive robot arm with two degrees of freedom moving in the vertical plane (see Figure 2) [18].

The vision system consists of a Panasonic GPMF502 camera with a lens having a focal length $\lambda=0.008[\mathrm{~m}]$ and a DT3851-4 frame processor board from Data Translation. A black disc was mounted on the robot end-effector, the centroid of disc was selected as the object feature point. 


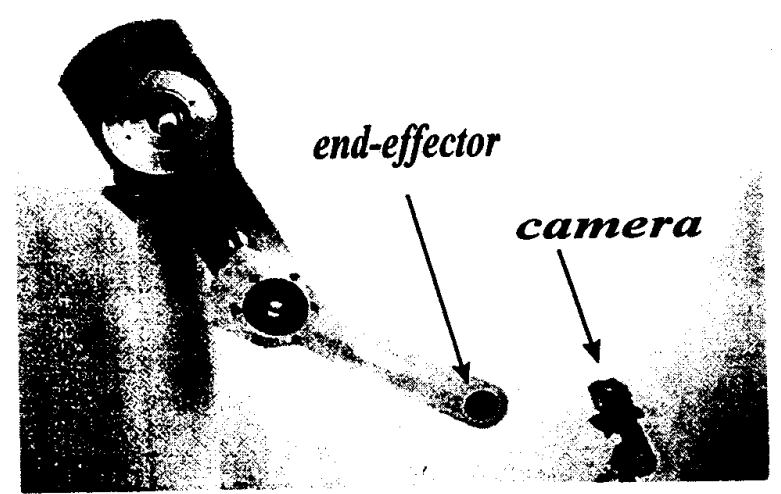

Figure 2: Robot arm

The CCD camera was placed in front of the robot arm at a distance $o_{C_{3}}=0.64[\mathrm{~m}]$ and rotation $\theta=0$ [degrees] with respect to the system $\Sigma_{R}$.

The controller (8)-(9) and (14)-(15) was written in $\mathrm{C}$ language and executed in the control board at 2.5 msec. sampling rate while the visual feedback loop was at $50 \mathrm{msec}$.

\section{Experimental results}

This section describes the experimental results obtained by testing the visual servo controller on the direct drive robot manipulator.

The desired motion of the image feature was set to

$$
\begin{array}{lll}
u_{d}(t) & =220+55 \sin (t) & \text { [pixels] } \\
v_{d}(t)=220+55 \cos (t) & \text { [pixels] }
\end{array}
$$

while the initial configuration of robot was $q_{1}(0)=$ 10 [degrees] and $q_{2}(0)=70$ [degrees], giving $[u(0) v(0)]^{T}=\left[\begin{array}{ll}262 & 81\end{array}\right]^{T}$ pixels and $\dot{\boldsymbol{q}}(0)=0$ [ degrees/sec].

Figures 3-4 shown the experimental results. Figure 3 depicts the time evolution of the feature error vector $\left[\begin{array}{lll}\tilde{u} & \tilde{v}\end{array}\right]^{T}$. After a smooth transient, both components of the feature position error tend asymptotically to a small neighborhood of zero.

The path that accomplishes the robot manipulator end-effector in the image plane $u-v$ is shown in figure 4. It can be observed that the end-effector evolves within a small neighborhood of the desired feature path. The oscillatory behavior may be due mainly to the friction at the joints and the delay in the image

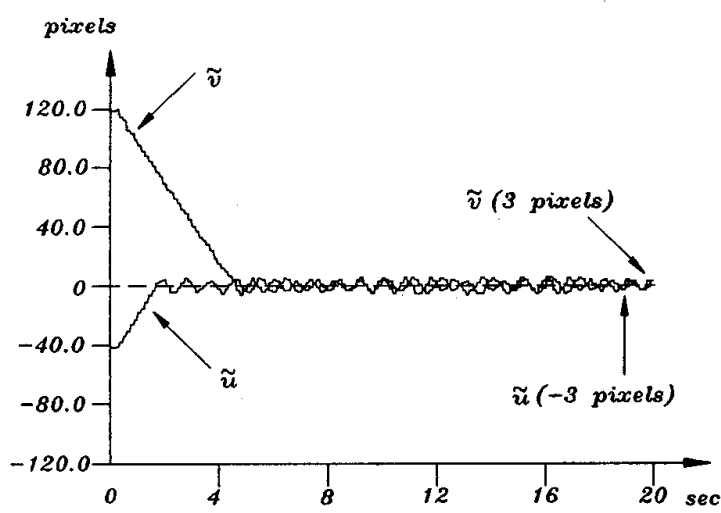

Figure 3: Components of image feature error

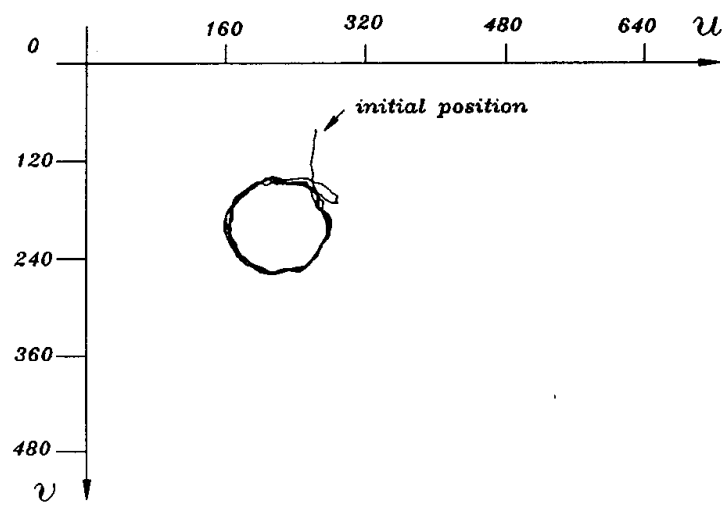

Figure 4: Path of the robot manipulator end-effector in the image plane

feature extraction, since the visual sampling period is 50 msec., this is, between two image samples the arm moves in "open loop" with respect to image errors.

From experimental results of this controller, we can note that despite of the feature position error is usually large in the initial transient, the hyperbolic function $\tanh (\cdot)$ indirectly limits the applied torques and produces a smooth response.

\section{Conclusions}

This paper has presented a two loops direct visual controller for fixed-camera robotic systems. This strategy is based on the look-and-move philosophy in the sense that an image-based outer loop is used to 
produce the desired robot velocity. However, the originality lie in the fact that an explicit velocity inner loop based on inverse dynamics is employed to drive the robot via the joint torques. The overall closed-loop system is shown to be locally asymptotically stable.

Implementation of the proposed control scheme requires the knowledge of the Jacobian $J(q)$ which in turn needs information on some intrinsic and extrinsic camera parameters.

The experimental evaluation on a direct drive arm showed good performance, however, the presence of friction in the joints as well as the slow video rate produce small oscillations of the image feature error.

\section{References}

[1] Hutchinson S., G.D. Hager, and P. I. Corke, "A tutorial on visual servo control", IEEE Transactions on Robotics and Automation, Vol. 12, No. 5, October 1996, pp. 651-670.

[2] Hashimoto K., Visual servoing, World Scientific Publishing, 1993.

[3] Koivo A. J. and N. Houshangi, "Real-time vision feedback for servoing robotic manipulators with self-tuning controller". IEEE Tran. Syst. Man, Cybern., Vol. 21, No. 1, 1991, pp. 134-142.

[4] Feddema J. T., C. S. G. Lee and O. R. Mitchell, "Weighted selection of image features for resolved rate visual feedback control", IEEE Transactions on Robotics and Automation, February 1991, Vol. 7, No. 1, pp. 31-47.

[5] Espiau B., F. Chaumette, and P. Rives, "A new approach to visual servoing in robotics", IEEE Trans. on Rob. and Aut., Vol. 8, No. 3, 1992, pp. 313-326.

[6] Wijesoma W. S., D. F. H. Wolfe and R. J. Richards, "Eye to hand coordination for visionguided robot control applications", The Int. J. Robot Res., Vol. 12, No. 1, 1993, pp. 65-78.

[7] Allen P. K., A. Timcenko, B. Yoshimi and P. Michelman, "Automated tracking and grasping of a moving object with a robotic hand-eye system", IEEE Trans. on Robotics and Automation, Vol. 9, No. 2, April 1993, pp. 152-165.

[8] Lei M. and B. K. Ghosh, "Visually guide robotic tracking and grasping of a moving object", Proc. 32nd. Conf. on Decision and Control, San Antonio, TX, Dec. 1993, pp. 1604-1609.
[9] Hager G. D., W. C. Chang, and A. S. Morse, "Robot hand-eye coordination based on stereo vision". IEEE Control System, Vol. 15, No. 1, February 1995, pp. 30-39.

[10] Nelson B. J., N. P. Papanikolopoulos and P. Khosla, "Robotic visual servoing and robotic assembly tasks", IEEE Robotics and Automation Magazine, Vol. 3, No. 2, June 1996, pp. 23-31.

[11] Miyazaki F. and Y. Masutani, "Robustness of sensory feedback control based on imperfect Jacobian", Robotics Research: The Fifth International Symposium, H. Miura and S. Arimoto, Eds. Cambridge, MA: MIT Press, 1990, pp. 201-208.

[12] Kelly. R., P. Shirkey and M. W. Spong, "Fixed camera visual servo control for planar robots". Proceedings of the IEEE International Conference Robotics and Automation, April 1996, pp. Minneapolis, MN., Vol. 3, pp. 2463-2469.

[13] Kelly R., "Robust asymptotically stable visual servoing of planar robots", IEEE Transactions on Robotics and Automation, Vol. 12, No. 5, October 1996, pp. 759-766.

[14] Maruyama A. and M. Fujita, "Robust visual servo control for planar manipulators with eyein-hand configurations", in Proc. of the IEEE Conference on Decision and Control, San Diego, CA., December 1997, pp. 2551-2552.

[15] Hager G.D., "A modular system for robust positioning using feedback from stereo vision", IEEE Trans. on Robotics and Automation, Vol. 13, No. 4, August 1997, pp. 582-595.

[16] Spong M. and M. Vidyasagar, "Robot Dynamics and Control”, John Wiley, New York, 1989.

[17] Hashimoto K. and H. Kimura, "LQ optimal and nonlinear approaches to visual servoing", in Visual Servoing, Edited by K. Hashimoto, World Scientific Pub., Singapore, 1993, pp. 165-198.

[18] Reyes F. and R. Kelly, "Experimental evaluation of identification schemes on a direct drive robot", Robotica, Vol. 15, Part 5, September-October 1997, pp. 563-571. 